\title{
EFFECT OF CENTELLA ASIATICA L. ON THE NUMBER OF OSTEOCLASTS, OSTEOBLASTS, AND OSTEOCYTES IN THE TIBIAE OF OVARIECTOMIZED RATS
}

\author{
VANISSA KARIS, SRI ANGKY SOEKANTO*, ERIK IDRUS
}

Department of Oral Biology, Faculty of Dentistry, Universitas Indonesia, Jakarta, 10430, Indonesia. Email: sasoekanto@gmail.com Received 17 October 2018, Revised and Accepted 27 December 2018

\author{
ABSTRACT \\ Objective: This study aimed to examine the effectiveness of Centella asiatica L. as a phytoestrogen. \\ Methods: Therapy using C. asiatica extract at doses of $60 \mathrm{mg} / \mathrm{kg}$ body weight, $120 \mathrm{mg} / \mathrm{kg} \mathrm{BW}$, and $180 \mathrm{mg} / \mathrm{kg}$ BW was administered to ovariectomized \\ rats for 30 days.
}

Results: Histological examination using hematoxylin and eosin staining demonstrated an increased number of osteocytes and a decreased number of osteoclasts, but there was no significant increase in osteoblast numbers.

Conclusion: The administration of the low doses of $C$. asiatica extract affects the number of osteocytes and osteoclasts but not osteoblasts in ovariectomized rats.

Keywords: Centella asiatica, Estrogen, Osteoporosis, Osteocytes, Osteoclasts, Osteoblasts.

(C) 2019 The Authors. Published by Innovare Academic Sciences Pvt Ltd. This is an open access article under the CC BY license (http://creativecommons. org/licenses/by/4. 0/) DOI: http://dx.doi.org/10.22159/ijap.2019.v11s1.AR171

\section{INTRODUCTION}

Osteoporosis is a bone disease characterized by the reduction in the mass density or volume of bones. Reduced mass density could lead to the reduction in the mechanical strength of bones such that they are more susceptible to fractures [1]. The incidence of osteoporosis has been associated with old age, particularly in postmenopausal women, and has become a major health problem even in developed countries. The bone remodeling process in postmenopausal women significantly increases and continues to increase for up to 40 years after the cessation of ovarian function, leading to progressive and continuous bone resorption. Increased bone activity is probably mediated through shorter osteoblast life and prolonged osteoclast life [2].

Hormone replacement is the most effective and only therapy that shows significant results in preventing the loss of bone density and reducing the risk of fractures [3]. Many women prefer to use phytoestrogens rather than estrogen for hormone replacement therapy due to the adverse effects of synthetic estrogen, such as increased risk of breast cancer and endometrial bleeding. Phytoestrogens are plant-derived chemicals that have a similar function to the estrogen hormone naturally produced in the body [4]. Numerous plants, including Centella asiatica L., have been reported to contain phytoestrogens. $C$. asiatica is a cosmopolitan plant, which indicates its widespread distribution, particularly in the tropics and subtropics. It grows well in places with sufficient sunlight as well as in shaded places with fertile but rather damp soil.

The phytochemical content of $C$. asiatica leaf constitutes terpenoids, flavonoids, olive oil, and amino acids [5]. The flavonoid groups in C. asiatica, which act as phytoestrogens, include quercetin, kaempferol, various glycosides, catechins, rutin, naringin, castilliferol, and castillicetin $[6,7]$.

Kaempferol, a flavonol derivative of the Kaempferia galanga L. rhizome, is a phytoestrogen that can exert osteogenic effects and can also be found in C. asiatica. The previous research has shown that kaempferol stimulates the osteogenic differentiation of osteoblasts in vitro through estrogen receptor signaling [8]. Moreover, quercetin and kaempferol show an inhibitory effect on bone resorption and mature osteoclast apoptosis [9]. Quercetin, which is also present in C. asiatica, shows a significant stimulatory effect on the proliferation and mineralization of rat osteoblasts, thus promoting the bone formation process [10]

Other flavonol groups contained in C. asiatica leaves have been shown to prolong osteoblasts life and increase alkaline phosphatase activity. Reportedly, catechin therapy reduces the production of cytokines that induce bone resorption and apoptosis in osteoblasts, resulting in the prevention of osteoporosis and inflammatory bone disease [11]. Naringin, another flavonoid found in C. asiatica, could reduce the number of osteoclasts formed through interleukin (IL)-1induced differentiation. Furthermore, naringin increases bone mass by suppressing osteoclast formation in rats, thus preventing bone diseases such as osteoporosis [12].

This evidence indicates that $C$. asiatica may prevent postmenopausal osteoporosis by reducing the number of osteoclasts and increasing the number of osteoblasts.

\section{METHODS}

The study protocol had been approved by the Dental Research Ethics Committee, Faculty of Dentistry, Universitas Indonesia. This was an experimental study using a post-test control group design. In total, 30 laboratory rats were randomly assigned to one of the six groups: Control rats (SHAM); untreated ovariectomized rats (negative control); ovariectomized rats administered $C$. asiatica leaf extract at three different doses (P1=60, P2=120, and P3=180 mg $/ \mathrm{kg}$ body weight $[\mathrm{BW}])$; and ovariectomized rats administered Vitamin E (positive control) for 30 days. Histological sections were stained with hematoxylin and eosin and were used to calculate the number of osteoclasts, osteoblasts, and osteocytes (Fig. 1a-c). Then, the data on osteoclast, osteoblast, and osteocyte count were analyzed by the Shapiro-Wilk normality test. A non-parametric Kruskal-Wallis test was used for the non-homogeneous, non-normal distributed data. The significant results of osteoclast and osteocyte data were then analyzed by a post hoc Mann-Whitney U-test. Data on the numbers of osteoblast 

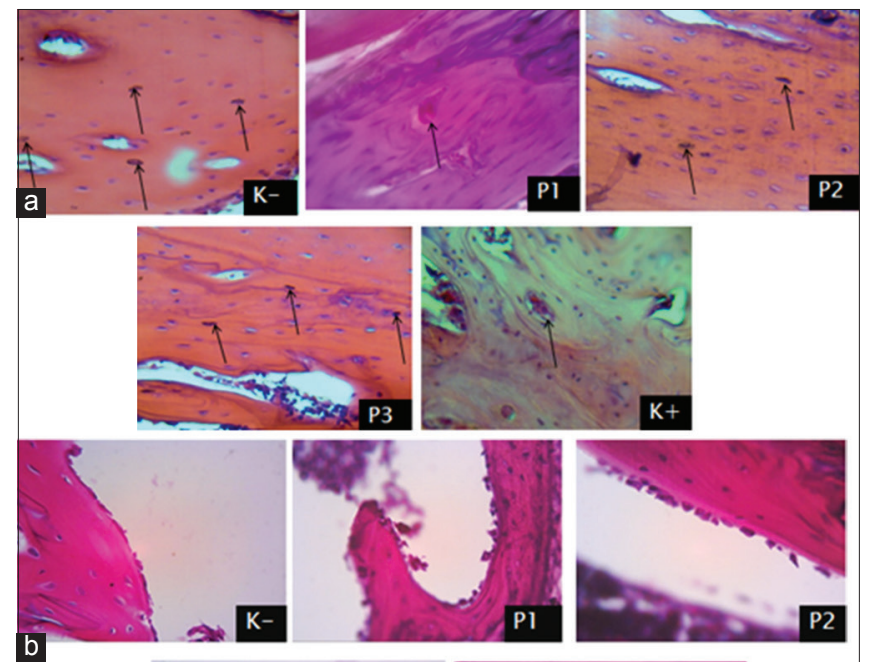

b
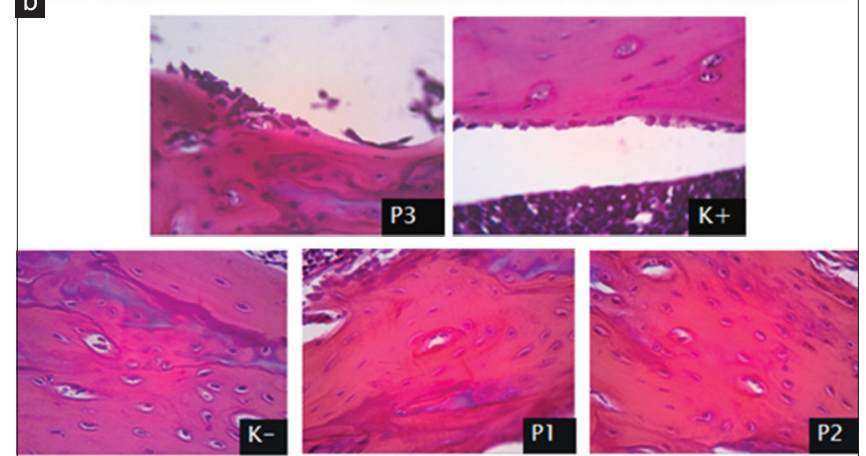

c
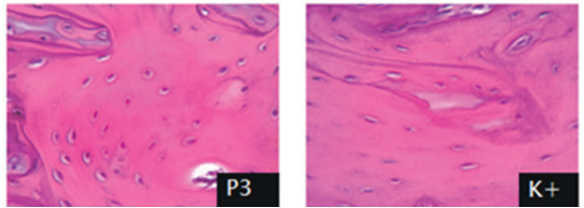

Fig. 1: (a) Histological appearance of osteoclasts in the tibia of rats (Stain: Hematoxylin and eosin), (b) Histological appearance of osteoblasts in the tibia of rats (Stain: Hematoxylin and eosin)

(c) Histological appearance of osteocytes in the tibia of rats (Stain: Hematoxylin and eosin)

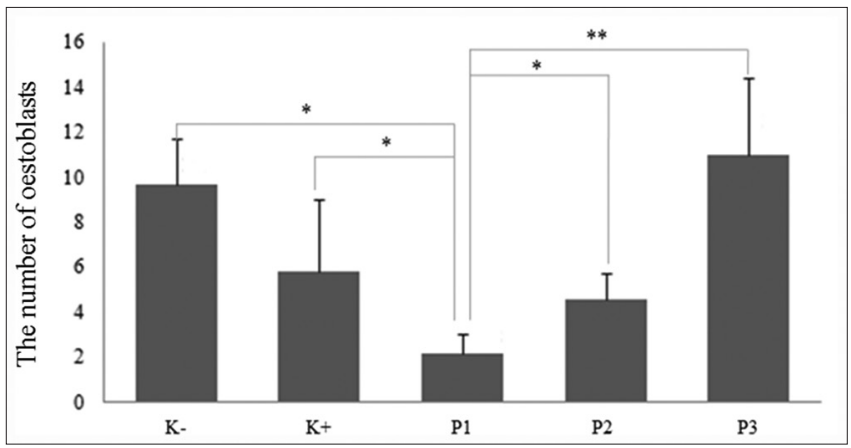

Fig. 2: Average and standard deviation of the number of osteoclasts in rats

and osteoclast were analyzed by Pearson's correlation test, and data on the number of osteocytes were analyzed by Spearman's correlation test.

\section{RESULTS}

The success of ovariectomy was determined by measuring the trabecular bone length of the SHAM and ovariectomized rats. In the same broad

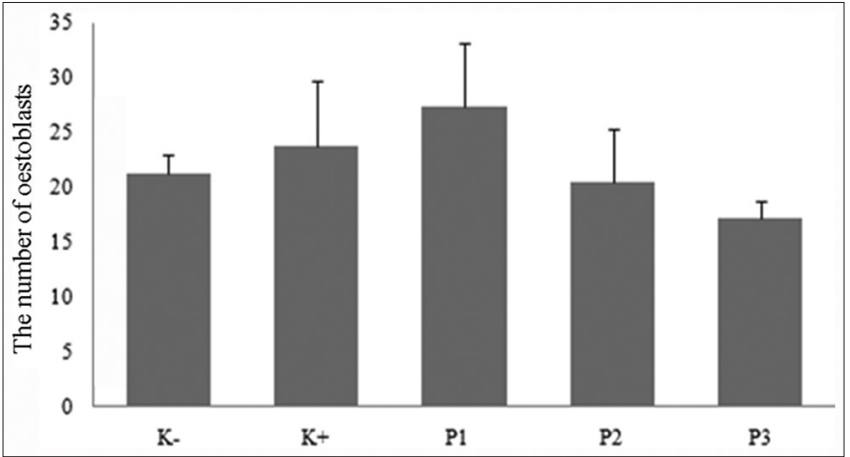

Fig. 3: Average and standard deviation of the number of osteoblasts in rats

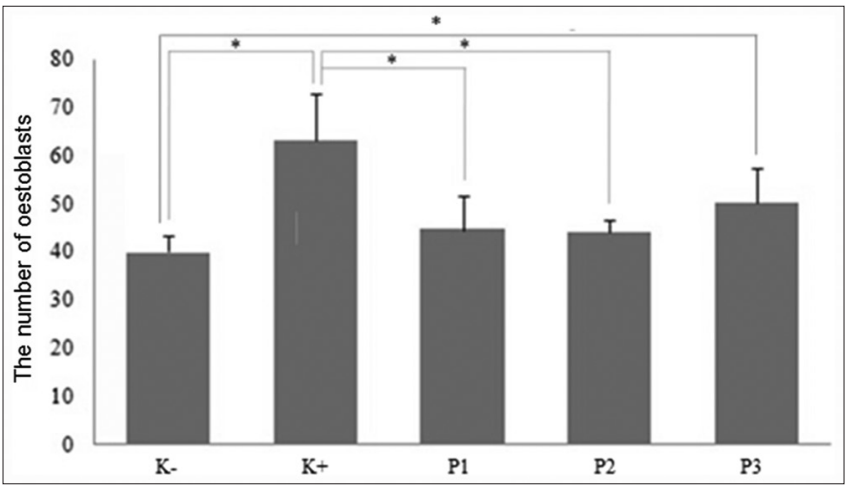

Fig. 4: Average and standard deviation of the number of osteocytes in rats

field of view, the length of the trabecular bone in unovariectomized rats was longer than that in the ovariectomized rats, indicating the effectiveness of ovariectomy procedure.

K-: Negative control group (aquadest); K+: Positive control group (Vitamin E); P1: Treated Group 1 (Centella asiatica, $60 \mathrm{mg} / \mathrm{kg} \mathrm{BW);}$ P2: Treated Group 2 (C. asiatica, $120 \mathrm{mg} / \mathrm{kg} \mathrm{BW);} \mathrm{P3:} \mathrm{Treated} \mathrm{Group} 3$ (C. asiatica, $180 \mathrm{mg} / \mathrm{kg} \mathrm{BW}$ ); ${ }^{*} \mathrm{p}<0.05$.

The results showed a significant decrease in the number of osteoclasts in the group administered with C. asiatica extract at doses of $60 \mathrm{mg} / \mathrm{kg} \mathrm{BW}$ (P1) and $120 \mathrm{mg} / \mathrm{kg} \mathrm{BW} \mathrm{(P2)} \mathrm{against} \mathrm{negative} \mathrm{control} \mathrm{(p<0.005)} \mathrm{(Fig.} \mathrm{2).}$

K-: Negative control group (aquadest); K+: Positive control group (Vitamin E); P1: Treated Group 1 (Centella asiatica, $60 \mathrm{mg} / \mathrm{kg} \mathrm{BW}$ ); P2: Treated Group 2 (C. asiatica, $120 \mathrm{mg} / \mathrm{kg}$ BW); P3: Treated Group 3 (C. asiatica, $180 \mathrm{mg} / \mathrm{kg} \mathrm{BW}$ ).

The results showed no significant increase in the number of osteoblasts (Fig. 3). However, there was a significant increase in the number of osteocytes in the negative control group (K-) administered with C. asiatica extract at a dose of $180 \mathrm{mg} / \mathrm{kg} \mathrm{BW}(\mathrm{p}<0.005)$, and a strong and significant correlation was observed between the administration of $C$. asiatica leaf extract and increase in the number of osteocytes ( $\mathrm{p}<0.005 ; \mathrm{R}=0.756$ ) (Fig. 4).

$\mathrm{K}-$ : Negative control group (aquadest); K+: Positive control group (Vitamin E); P1: Treated Group 1 (Centella asiatica, $60 \mathrm{mg} / \mathrm{kg} \mathrm{BW}$ ); P2: Treated Group 2 (C. asiatica, $120 \mathrm{mg} / \mathrm{kg} \mathrm{BW);} \mathrm{P3:} \mathrm{Treated} \mathrm{Group} 3$ (C. asiatica, $180 \mathrm{mg} / \mathrm{kg} \mathrm{BW}$ ); ${ }^{*} \mathrm{p}<0.05$.

\section{DISCUSSION}

Estrogens protect bones by inducing apoptosis in osteoclasts to balance the bone formation process by osteoblasts and bone resorption by 
osteoclasts. Many studies have shown that the Fas ligand (FasL) receptor plays a role in the process of estrogen-induced osteoclast apoptosis through the autocrine activation of the osteoclasts. FasL binds estrogen receptor $\alpha$ in osteoclasts, mediating the apoptosis of mature osteoclasts [13]. Other theories have posited the presence of paracrine mechanisms through which estrogen affects the survival of osteoclasts by increasing FasL in osteoblasts and triggering preosteoclast apoptosis [14].

Reportedly, flavonoids contained in C. asiatica initiate bone formation and inhibit bone resorption in vitro. Quercetin stimulates osteoblast proliferation and osteoclasts apoptosis. Naringin suppresses osteoclast number by inhibiting the production of IL-1 cytokine. Kaempferol stimulates the osteogenic differentiation of osteoblasts and apoptosis of osteoclasts, and catechins inhibit the production of tumor necrosis factor- $\alpha$ (TNF- $\alpha$ ) and IL- 6 cytokines, inhibiting osteoblast apoptosis. These findings suggest that $C$. asiatica can be effective in preventing osteoporosis.

Ovariectomy results in the increased production of TNF- $\alpha$ cytokines, which mediate osteoclastogenesis induced by receptor activator of nuclear factor kappa-B ligand and macrophage colony-stimulating factor, leading to increased osteoclast numbers [15]. Our findings demonstrated a significantly reduced osteoclast number in ovariectomized rats administered $C$. asiatica leaf extract at doses of $60 \mathrm{mg} / \mathrm{kg} \mathrm{BW}$ and $120 \mathrm{mg} / \mathrm{kg}$ BW but not at a dose of $180 \mathrm{mg} / \mathrm{kg} \mathrm{BW}$. The increased osteoclast number in the groups administered a dose of $180 \mathrm{mg} / \mathrm{kg}$ BW over 30 days indicates that this dosage of C. asiatica as a natural source of estrogen is not optimal to inhibit osteoclast differentiation despite increased osteoclast numbers. At very high doses, flavonoids function as pro-oxidants and allow the accumulation of free radicals within the body, thereby increasing oxidative stress that causes bone damage [16].

Estrogen deficiency stimulates osteoblast apoptosis and mediates oxidative stress in bones and bone marrow, thus accelerating bone loss [17]. Reportedly, C. asiatica exerts a protective effect against oxidative stress by activating the $\mathrm{d}$-aminolevulinic acid dehydratase enzyme, inhibiting lipid peroxidation, and activating antioxidant enzymes. Therefore, the use of $C$. asiatica could prevent osteoblast apoptosis, facilitating bone formation. However, C. asiatica therapy in this study did not lead to a significant increase in the number of osteoblasts in ovariectomized rats, which may be attributed to the short duration of the study. The dose-dependent properties of phytoestrogens may be another reason for this finding [18]. Other studies of catechin administration in ovariectomized rats or 3 months have reported increased bone density through increased osteoblast activity [19]. Phytoestrogens exert a dose-dependent effect on osteoblasts and osteoprogenitor cells, stimulating osteogenesis at low concentrations while inhibiting it at high doses [20]. Wattel [18] has reported that quercetin and kaempferol flavonoids optimally worked at varying doses. Various flavonoids are present in C. asiatica, such as quercetin, rutin, naringin, kaempferol, and catechins, but not all are effective due to their dose-dependent properties. Each type of phytoestrogen has a complex and specific action at different optimal doses [21]. Osteoclasts may have shown significant results because they take only 3 weeks to complete bone resorption, and many studies have shown that phytoestrogens function by inhibiting osteoclast formation and activity [20].

The results of statistical analysis showed that the correlation between increased $C$. asiatica leaf extract dose and osteocyte number in Wistar rats was strong and significant. The correlation direction was positive, indicating that greater the dose of $C$. asiatica leaf extract, greater would be the number of osteocytes in Wistar rats. Perhaps, increased osteocyte numbers maintain systemic mineral homeostasis in the body. Apparently, the ability to modify osteocyte formation facilitates the regulation of osteoblast function in bone disease, particularly osteoporosis. In addition, the increased number of osteocytes, increased osteogenesis by osteoblasts was expected in this study. The previous research has shown that strategies targeting the regulation of osteocyte function and its effect on osteoblast cell behavior are effective in reversing the process of decrease in bone mass with aging, thereby controlling osteogenic processes [22]. Therefore, angiotensin is expected to promote osteogenesis and helps to prevent osteoporosis.

The pharmacokinetics of phytoestrogens from $C$. asiatica could be influenced by other factors such as the duration of extract storage or the effect of other chemical constituents of $C$. asiatica on the rats that have not yet been studied. This proves that the administration of C. asiatica leaf extract at a dose of $180 \mathrm{mg} / \mathrm{kg}$ BW could increase the number of osteocytes in the tibia of ovariectomized Wistar rats at risk of osteoporosis.

Finally, C. asiatica extract administration could reduce the number of osteoclasts formed during the process of tibia bone destruction in rats due to the action of flavonoids such as the phytoestrogens and antioxidants, which can reduce oxidative stress. However, the extract administration did not increase the number of osteoblasts. Further, research is warranted to determine whether different doses of $C$. asiatica leaf extract can confer a preventive effect against osteoporosis in a larger patient cohort over a longer study duration ( $>3$ months).

Natural products offer a rich source of structurally diverse substances with a wide range of biological activities, which could be useful for the development of alternative or adjunctive for osteoporosis therapies [23]. The market for anti-aging products is also growing gradually, and customers are aware of active principles in the cosmetics [24]. C. asiatica as natural product also can be an alternative treatment for osteoporosis in aging women.

\section{CONCLUSION}

This experimental study demonstrated that the administration of C. asiatica leaf extract at doses of $60 \mathrm{mg} / \mathrm{kg} \mathrm{BW}$ and $120 \mathrm{mg} / \mathrm{kg} \mathrm{BW}$ significantly decreased the number of osteoclasts in ovariectomized rats. However, the administration of $C$. asiatica leaf extract did not significantly increase the number of osteoblasts. Furthermore, the administration of $C$. asiatica leaf extract at a dose of $180 \mathrm{mg} / \mathrm{kg}$ BW significantly increased the number of osteocytes following ovariectomy.

\section{CONFLICTS OF INTEREST}

There are no conflicts of interest to declare.

\section{REFERENCES}

1. Glaser DL, Kaplan FS. Osteoporosis. Definition and clinical presentation. Spine (Phila Pa 1976) 1997;22:12S-16S.

2. Martono B, Ghulamahdi M, Darusman LK, Aziz SA, Bermawie N. Criteria for markers of cultivation and asiaticoside productivity selection of Centella asiatica L. J Littr 2010;16:12-9.

3. Ismal NM. Postmenopausal osteoporosis: Epidemiology, pathophysiology and treatment. Malays J Pathol 1997;19:21-5.

4. Schart D. Phytoestrogens for menopausal. Nutr Action 2000;84:717.

5. Barnes J, Anderson L, Phillipson J. Herbal medicines. Pharm Press 2007:3:71-3.

6. Zheng CJ, Qin LP. Chemical components of Centella asiatica and their bioactivities. Zhong Xi Yi Jie He Xue Bao 2007;5:348-51.

7. Subban R, Veerakumar A, Manimaran R, Hashim KM, Balachandran I. Two new flavonoids from Centella asiatica (Linn.). J Nat Med 2008;62:369-73.

8. Lane NE. Epidemiology, etiology, and diagnosis of osteoporosis. Am J Obstet Gynecol 2006;194:S3-11.

9. Guo AJ, Choi RC, Zheng KY, Chen VP, Dong TT, Wang ZT, et al. Kaempferol as a flavonoid induces osteoblastic differentiation via estrogen receptor signaling. Chin Med 2012;7:10.

10. Endang L. Phytoestrogens: Safe Natural Compounds as a Substitute for Estrogen Hormones in Women. Yogyakarta: Universitas Gajah Mada; 2011.

11. Choi EM, Hwang JK. Effects of $(+)$-catechin on the function of osteoblastic cells. Biol Pharm Bull 2003;26:523-6.

12. Yang YJ, Yang ZL, Wang DC, Xiao XC, Li P. Comparative study on effects of rutin and quercetin on metabolism in osteoblast cells. Zhong Yao Cai 2006;29:467-70. 
13. Nakamura T, Imai Y, Matsumoto T, Sato S, Takeuchi K, Igarashi K, et al. Estrogen prevents bone loss via estrogen receptor alpha and induction of fas ligand in osteoclasts. Cell 2007;130:811-23.

14. Krum SA, Miranda-Carboni GA, Hauschka PV, Carroll JS, Lane TF, Freedman LP, et al. Estrogen protects bone by inducing fas ligand in osteoblasts to regulate osteoclast survival. EMBO J 2008;27:535-45.

15. Cenci S, Weitzmann MN, Roggia C, Namba N, Novack D, Woodring J, et al. Estrogen deficiency induces bone loss by enhancing T-cell production of TNF-alpha. J Clin Invest 2000;106:1229-37.

16. Skibola CF, Smith MT. Potential health impacts of excessive flavonoid intake. Free Radic Biol Med 2000;29:375-83.

17. Almeida M, Martin-Millan M, Ambrogini E, Bradsher $\mathrm{R} 3^{\text {rd }}$ Han L, Chen XD, et al. Estrogens attenuate oxidative stress and the differentiation and apoptosis of osteoblasts by DNA-bindingindependent actions of the ERalpha. J Bone Miner Res 2010;25:769-81.

18. Wattel A, Kamel S, Mentaverri R, Lorget F, Prouillet C, Petit JP, et al. Potent inhibitory effect of naturally occurring flavonoids quercetin and kaempferol on in vitro osteoclastic bone resorption. Biochem
Pharmacol 2003;65:35-42.

19. Bakhsh A, Mustapha NM, Mohamed S. Catechin-rich oil palm leaf extract enhances bone calcium content of estrogen-deficient rats. Nutrition 2013;29:667-72.

20. Dang ZC, Lowik C. Dose-dependent effects of phytoestrogens on bone. Trends Endocrinol Metab 2005;16:207-13.

21. Umland EM, Cauffield JS, Kirk JK, Thomason TE. Phytoestrogens as therapeutic alternatives to traditional hormone replacement in postmenopausal women. Pharmacotherapy 2000;20:981-90.

22. Graham JM, Ayati BP, Holstein SA, Martin JA. The Role of Osteocytes in Targeted Bone Remodeling: A Mathematical Model 2013; 8:5e63884.

23. Rakhman L, Pangestika O, Fitriani D, Rudhanton R, Permatasari N. Aloe vera ethanol extract as a therapy for alveolar mandible regeneration in lipopolysaccharide-exposed rats. 2018;10:11-4.

24. Sivamaruthi B, Kesika P. Chaiyasut C. A Review on anti-aging properties of probiotics. International Journal of Applied Pharmaceutics 2018;10(5):23-7. 